\section{Der DRK-Qualitätsstandard Kinderschutz}

Damit Kinderschutz in DRK-Kitas nicht dem Zufall überlassen ist, hat »eine Arbeitsgruppe aus Experten des DRKLandesverbandes Niedersachsen und der Landesstelle Jugendschutz mit unterschiedlichen Erfahrungshintergründen unter Leitung des DRK-Landesverbandes Niedersachsen e. V. einen Qualitätsstan- dard Kinderschutz erarbeitet. Nach einer Erprobungsphase in mehreren Kitas wird der Qualitätsstandard jetzt allen DRKKitas im Bereich des DRK-Landesverbandes Niedersachsen e. V. zur Anwendung empfohlen." (DRK-Landesverband Niedersachsen: Qualitätsstandard Kinderschutz, S. 5). Im Einzelnen werden folgende Themenbereiche beschrieben:

- Qualifikation der pädagogischen Fachkräfte
- Risikoeinschätzung

- Einbeziehung der »insoweit erfahrenen Fachkraft "

- Beratung der Eltern

- Maßnahmen zum Kinderschutz

- Einbeziehung des Jugendamtes

Ziel des Qualitätsstandards ist es, durch das frühzeitige Erkennen von Verdachtsfällen geeignete Maßnahmen einzuleiten, die die Folgen von Kindeswohlgefährdung minimieren. Dazu zählen Beratung, Unter-

\title{
Kinderschutz in der Praxis
}

Ein Erfahrungsbericht aus dem DRK-Kreisverband Hameln-Pyrmont

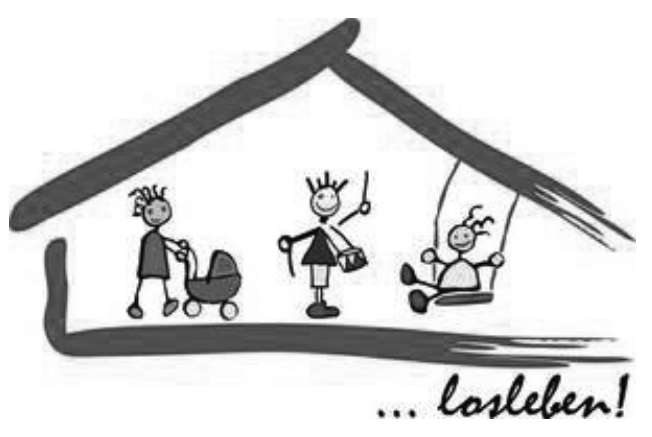

Alles fing damit an, dass zwei Erzieherinnen aus der DRKKita »Neue Straße « in Emmerthal im Jahre 1996 begonnen hatten, sich mit dem Thema Missbrauch und sexualisierte Gewalt an Kindern auseinanderzusetzen. Ein schwerer Brocken! Aber wir tauchten immer mehr in die Materie ein, was nicht zuletzt an den guten Fortbildungsreferentinnen lag. Genau das war unser Thema. Und uns war klar: Daraus muss mehr werden. Jedes dritte bis vierte Mädchen und jeder fünfte bis sechste Junge sind sexueller Gewalt ausgesetzt (und die Dunkelziffer liegt um ein Vielfaches höher!).

Diese erschreckenden Zahlen waren und sind unser Antrieb, aktiv zu werden und es auch zu bleiben. In den meisten Fällen sind Familienangehörige oder Vertrauenspersonen die Täter und Täterinnen, nicht wie allgemein angenommen völlig fremde Personen. Das bestätigen auch die aktuellen Berichterstattungen in den Medien.

Dann ging alles ganz schnell: Das Team, die Leitung, die Fachberatung, die anderen DRK-Kitas in unserem Kreisverband und die Geschäftsstelle, alle zogen mit - bis heute. Daraus hat sich wertvolle, spannende und motivierende Kinderschutzarbeit entwickelt. Einen richtigen Schub haben wir nochmals bekommen, als das SGB VIII 2005 hinsichtlich des Umgangs mit Kindeswohlgefährdung konkretisiert und erweitert wurde.

Viele fachliche Institutionen (Kinderschutzbund Hameln, Landesstelle Jugendschutz Niedersachsen, DRK-Landesverband) haben uns bestätigt, dass wir uns auf dem richtigen
Weg befinden, was uns ermutigte, weiterzumachen. Die Vorgabe aus dem Qualitätsstandard des DRK-Landesverbandes Niedersachsen e. V. »Jede Kindertageseinrichtung berücksichtigt in ihren Bildungs- und Erziehungszielen das Kindeswohl und trifft hierzu Festlegungen « fällt den DRK-Kitas im Kreisverband Hameln-Pyrmont daher nicht schwer umzusetzen.

Bereits 1998 haben wir begonnen in unseren Einrichtungen konkret zum Thema Kinderschutz zu arbeiten. Im Vordergrund stand damals, das pädagogische Fachpersonal kompetent zu schulen und auf diese Aufgabe vorzubereiten. Das passierte sowohl durch kreisverbandsinterne als auch überregionale Fortbildungen. Wir wollten kein einmaliges Programm oder Projekt absolvieren, sondern langfristig erfolgreiche Präventionsarbeit leisten. Inhalte sollten handlungsorientiert und ganzheitlich umgesetzt und Arbeitsformen gewählt werden, in denen die Kinder selbst aktiv werden können (z. B. in Rollenspielen).

Darüber hinaus waren altersspezifische, interkulturelle und geschlechtsspezifische Angebote genauso wichtig, wie das zeitweise Arbeiten in altershomogenen Gruppen. Nicht zuletzt war es wichtig, das gesamte Kita-Team und die Eltern in das Präventionskonzept einzubeziehen. Besonders die Eltern bilden einen wichtigen Bestandteil präventiver Arbeit und sollen jederzeit bestens über die Inhalte der Projekte informiert sein und gerne mit »ins Boot geholt « werden. Denn auch bei Eltern bestehen Unsicherheiten, wenn es zum Beispiel um das Thema Sexualpädagogik geht. 
stützung der Familien, Vermittlung von Hilfen und Kooperation mit anderen Beteiligten. (DRK-Landesverband Niedersachsen: Qualitätsstandard Kinderschutz, S. 6)

Da der Qualitätsstandard Anforderungen des Qualitätsmanagements nach DIN EN ISO 9000 f erfüllt, kann er in bestehende oder noch zu erarbeitende Qualitätshandbücher übernommen werden.

In unserem langjährigen Arbeitskreis Prävention haben wir für die Erzieherinnen Strategien entwickelt, um sich unter fachkundiger Leitung mit dem Thema Kindeswohlgefährdung im Allgemeinen und sexualisierter Gewalt im Speziellen auseinanderzusetzen. Natürlich gab und gibt es Unsicherheiten, die wir durch kompetente und konsequente Auseinandersetzung mit dem Thema mindern und weitestgehend zerstreuen konnten und können. Inzwischen ist unser Präventionsprojekt »Starke Kinder sagen Nein! « zum festen Bestandteil unserer pädagogischen Arbeit geworden und wir haben dabei nicht nur die Kinder und ihre Familien im Blick, sondern auch unser Umfeld. Wir signalisieren: Wir sehen hin! Für uns ist sexualisierte Gewalt an Kindern kein Tabu. Wir reden darüber und wir handeln! Dabei liegt unser Schwerpunkt ganz klar auf Vorbeugung und setzt erst im nächsten Schritt auf Intervention. Der Qualitätsstandard Kinderschutz des DRK-Landesverbandes Niedersachsen e. V. ist dabei eine gute Unterstützung.

Bausteine unseres Präventionsprojektes »Starke Kinder sagen Nein! « sind

- Sexualpädagogik

- Bestimmungsrecht über den eigenen Körper

- Wahrnehmung von Gefühlen, Vertrauen auf die eigene Intuition

- Unterscheidung zwischen guten, schlechten, komischen und verwirrenden Berührungen

- Umgang mit Geheimnissen

- Nein-Sagen-dürfen und Ja-Sagen-können

In unserer pädagogischen Arbeit setzen wir die Bausteine in der Form um, dass wir die Selbstbildungsprozesse der Kinder unterstützen, indem wir sie zum selbständigen Lernen herausfordern. In allen Präventionsbausteinen geben wir jedem einzelnen Kind die Möglichkeit sich mit der Thematik und sich selbst, seinem Tempo und Entwicklungsstand entsprechend, auseinanderzusetzen. Ziel dabei ist es,

- Mädchen und Jungen in ihren Handlungskompetenzen zu stärken

- dass Mädchen und Jungen ihre persönlichen Kraftquellen und Ressourcen entdecken, um daraus Energie und Mut zu schöpfen und damit
- die Kinder in ihrem Selbstbewusstsein und Selbstwertgefühl zu stärken, ganz nach der Devise »Hilf mir, es selbst zu tun!«, bzw. »Hilf mir, ich selbst zu sein!«

Wir bieten uns dabei aktiv als Bindungsperson an, die Sicherheit und Geborgenheit vermittelt. Kindliche Bildungsprozesse gelingen nur, wenn sie in gute Beziehungen eingebettet sind. Bindung ist eine elementare Voraussetzung für Bildung. In diesem Sinne verstehen wir Präventionsarbeit als Bildungsarbeit, die mit all ihren Elementen in den Kitaalltag integriert ist. Im Verlaufe dieses Prozesses sind auch wir Erzieherinnen sensibler geworden die Kinder in ihren Selbstbildungsprozessen zu unterstützen und achten noch mehr die Eigenständigkeit und individuelle Persönlichkeit jedes Einzelnen. In diesem $\mathrm{Zu}$ sammenhang ist uns bewusst geworden, dass Kinderschutz sehr viel mit eigenen Einstellungen und Haltungen zu tun hat. Von daher hinterfragen wir unsere eigenen Verhaltens- und Denkweisen mit Hilfe von Selbst- und Fremdreflexion immer wieder neu und schauen dabei auch auf die eigene Bildungsbiografie.

Über die pädagogische Arbeit hinaus ist es im Rahmen unserer Kinderschutzaktivitäten unser Ziel kompetent zu informieren, zu motivieren hinzusehen, aufzustehen und die Stimme zu erheben, wenn es geboten ist und somit einen wichtigen Beitrag zum Schutz der Kinder in unserer Gesellschaft zu leisten. Denn ein informiertes und handlungsfähiges Umfeld bietet den besten Schutz für unsere Kinder. Damit das gelingt, findet in den DRK-Kitas des Kreisverbandes Hameln-Pyrmont nicht nur jährlich unser Projekt »Starke Kinder sagen Nein! «statt, sondern auch regelmäßige Aktionswochen mit unterschiedlichen Schwerpunkten, die einer breiten Öffentlichkeit zugänglich sind.

Rückblickend stellen wir fest, dass die Kinder in unseren Kitas immer selbstbewusster werden und die Eltern unsere Arbeit begrüßen und unterstützen. Starke und selbstbewusste Kinder sind anstrengend und fordern uns heraus, aber sie sind auch unbequeme Opfer und deshalb besser vor Übergriffen geschützt. Kinderschutz ist mit dem Projekt »Starke Kinder sagen Nein! « daher eines unserer wichtigsten und beliebtesten Projekte!

Indra Korgol ist Erzieherin und Expertin zum Thema »Prävention von sexualisierter Gewalt an Mädchen und Jungen «. 
Kind: "Heute Essen?"

Erzieherin: „Heute gibt' es Spaghetti.

Aber sage das noch mal richtig:

"Was gibt es heute zu essen?ı"

Kind: "Spaghetti."

Dialog im Kindergarten

"Viele Kinder sind heute Waisen mit Eltern." Margarete Mitscherlich, deutsche Psychoanalytikerin (geb. 1917)

"Wenn die Ohrfeige keinem schadet, warum führen wir sie nicht unter Erwachsenen ein?"

Dorle Marx, deutsche SPD-Politikerin und Juristin

"Holt die Kinder aus den Heimen: Ja, wenn Ihr etwas Besseres habt.

Dann ganz sicher. Aber nur dann." Andreas Mehringer, deutscher Sozialpädagoge (1911-2004)

„Alle glücklichen Familien gleichen einander, jede unglückliche Familie ist auf ihre eigene Art unglücklich." Lew Tolstoi, russischer Schriftsteller (1828-1910)

"Alle schlechten Angewohnheiten entwickeln sich in der Familie. Das fängt mit Mord an und geht über Betrug und Trunksucht manchmal bis zum Rauchen." Alfred Hitchcock, britischer Regisseur (1899-1980)

"Heiraten, eine Familie gründen, alle Kinder, welche kommen, hinnehmen, in dieser unsicheren Welt erhalten und gar ein wenig führen, ist meiner Überzeugung nach das Äußerste, das einem Menschen überhaupt gelingen kann."

Franz Kafka, österreichischer Schriftsteller (1883-1924) 